\title{
Efficacy of Sympathetic Radiofrequency in CRPS 1 Satellite Ganglion Block VS T2-T3 Sympathetic Block
}

\author{
Article by Jayesh Thakrar \\ Ph.D in Medicine by Research in Anesthesiology, Texila American University, India \\ Email:dr_thakrarjayesh@yahoo.co.in
}

\begin{abstract}
Background: This study is performed to evaluate the treatment of complex regional pain syndrome CRPS type 1 with radiofrequency thermo coagulation of stellate ganglion block and T2-T3 sympathetic block.

Material and methods: We performed diagnostic stellate ganglion block in all patient with CRPS TYPE 1 in one hand. Those patients reduce 50\% in pain score e.g. VAS SCORE, was considered for RF stelleate ganglion block after 1 week and this is GROUP 1. Those patients reduce less than 50\% pain score was considered for diagnostic T2-T3 sympathetic block after 1 week. Those patients reduce 50\% in pain score e.g. VAS SCORE, was considered for RF T2-T3 sympathetic block after 1 week and this is GROUP 2. Pain intensity using a visual analog score also assessed usage of analgesic dosages before and after RF in both groups.

Results: Treatment produced a significant decrease in pain score, VAS value in both groups and significant reduce the dosages of analgesic drugs in both groups.

Conclusion: The study of these 17 patients shows that there is chance of inadequate pain relief after satisfactory stellate ganglion block because of KUNTZ'S nerve and giving T2-T3 sympathetic block among this gives good pain relief. Also RF in stellate ganglion and T2-T3 sympathetic ganglion block gives long term pain relief and decrease analgesic dosages and thus improves quality of life.
\end{abstract}

Keywords: Complex regional pain syndrome type 1, Stellate ganglion block, T2-T3 sympathetic block, RF-radiofrequency, VAS score, analgesic drugs

\section{Introduction}

Complex Regional Pain syndrome (CRPS) 1 arises following trauma to a limb and is characterized by functional impairment in the affected body segment. It is associated with intense sensory, autonomic, motor and trophic changes, which are disproportionate to the inciting event and cannot be accounted for by other causes of chronic pain (1). Despite recent advances in the understanding of its pathophysiology, pain relief in CRPS remains a major challenge. This is partly due to the complexity of the mechanism underlying the maintenance of pain and the functional impairment present in this syndrome, but it is also related to the lack of evidence-based treatment trial specific for this condition (2). Most interventions used for CRPS relief are not supported by high quality evidence-based data (3). It is mainly diagnosed by IASP criteria (11)

\section{IASP diagnostic criteria for CRPS}

- The presence of an initiating noxious event or a cause of immobilization.

- Continuing pain, allodynia or hyperalgesia with which the pain is disproportionate to any inciting event.

- Evidence at some time of edema, changes in skin blood flow, or abnormal sudomotor activity in the region of pain.

- This diagnosis is excluded by the existence of conditions that would otherwise account for the degree of pain and dysfunction. 
Texila International Journal of Medicine

Volume 4, Issue 2, Dec 2016

- TYPE I: Without obvious nerve damage $\{$ aka RSD $\}$.

- TYPE II: With obvious nerve damage \{aka Causalgia\}.

\section{Budapest diagnostic criteria for CRPS}

\begin{tabular}{|l}
\hline General Definition of the Syndrome \\
\hline CRPS describes an array of painful conditions that are characterized by a continuing (spontaneous \\
and/or evoked) regional pain that is seemingly disproportionate in the time or degree to the usual \\
course of any known trauma or other lesion. The pain is regional (not in a specific nerve territory or \\
dermatome), but may spread, and usually has a distal predominance of abnormal sensory, motor, \\
sudomotor, vasomotor, and/or trophic findings. The syndrome show variable progression over time. \\
\hline To make clinical diagnosis, the following criteria must be met: \\
- Continuing pain, which is disproportionate to any inciting event. \\
- Must report at least one symptoms in 'three of the four' following categories: \\
- SENSORY: Reports of hyperalgesia and/or allodynia. \\
VASOMOTOR: Reports of temperature asymmetry and/or skin color changes and/or \\
- $\quad$ Skin color asymmetry. \\
- $\quad$ asymmetry. \\
MOTOR/TROPHIC: Reports of decreased range of motion and/or motor dysfunction \\
(weakness, tremor, dystonia) and/or trophic changes (hair, nail, skin). \\
Must display at least one sing at time of evaluation in two or more of the following \\
categories: \\
- SENSORY: Evidence of hyperalgesia (to pinprick) and/or allodynia (to light touch \\
and/or deep somatic pressure and/or joint movement). \\
VASOMOTOR: Evidence of temperature asymmetry and/or skin color changes and/or \\
skin color asymmetry. \\
- SUDOMOTR/EDEMA: Evidence of edema and/or sweating changes and/or sweating \\
asymmetry. \\
MOTOR/TROPHIC: Evidence of decreased range of motion and/or motor dysfunction \\
(weakness, tremor, dystonia) and/or trophic changes (hair, nail, skin).
\end{tabular}

According to Sandroni incidence of CRPS I 5.46 new cases/100000 annually (18). According to IASP incidence is 25.2 new cases/100000 annually (18). Period prevalence is 20.57/100000 (18). Female: male was 4:1, mostly 50-70 year age with median age of 46 years at onset (19). Upper limb was affected twice as commonly as lower limb (19).

Even though the pathophysiology is not clearly defined (20). The syndrome may be mainly a systemic disease involving the central and peripheral nervous system, yet the specific interaction between the central and peripheral mechanisms is unclear 8. The postulated mechanisms includes: Inflammation, Afferent dysfunction, Central dysfunction \&Sympathetic dysfunction.

CRPS is diagnosed by IASP Diagnostic criteria for CRPS \& “Budapest” Diagnostic criteria (11)

Limited understanding of the pathophysiology mechanism involved in the develop of CRPS and absence of clear objective diagnostic criteria, multimodal, multidisciplinary approaches requires to treat CRPS (19). Effective pain control: (1) pharmacologic (2) interventional methods by IVRA, Sympathetic nerve blocks and Spinal cord stimulation, Functional restoration, Rehabilitation-Based Treatment Modalities, Psychologic Interventions \& Other Therapeutic Modalities (5, 6, 7, 19)

Sympathetic nerve block has been used for treatment of CRPS since the beginning of the $20^{\text {th }}$ century (4). The technique that is most commonly used to target sympathetic innervations of the upper limbs is the stellate ganglion block (6, 8, and 10). Anatomical and clinical studies have suggested that this may not the most effective technique for upper limb sympathetic block 
$(4,12,13)$. Second order neurone cell bodies that supply the upper limb are located in the intermediolateral horn of the thoracic spinal cord. Preganglionic fibers ascend cephalad and synapse on postganglionic fiber, primarily in the $2^{\text {nd }}$ (and to a lesser extent in the 3rd) thoracic sympathetic ganglia, before ascending and passing through the stellate and the middle cervical ganglia, en route to the upper limb $(14,15)$. However, in $20 \%$ of the individual, nerves from these two thoracic sympathetic ganglia projects directly to the brachial plexus, by passing the upper stellate and middle cervical ganglia $(14,15$, and 9). Thus different from SGB, which only influences nerve that actually passes through this structure before reaching the upper limb, TSB act directly on the main synapse site of most sympathetic fibers innervating this body segment(14,15\}. Despite this potential relevant anatomical information, TSB has rarely been evaluated in CRPS patients $(16,17)$.

\section{Methods}

This study is consisted of 15 patients attending to the Advance Pain Care Clinic, Surat, Gujarat, India from July 2015 to December 2015 with diagnosis of CRPS 1 in one hand.

The diagnosis is based on IASP-BUDAPEST criteria (11) with VAS $>4 / 10$ with failed conservative management (6weeks).

All 15 patients were examined and diagnosis of CRPS type 1, right side 8 and 7 left were included. 9 patients were women and 6 were men. Age of patients were between 18-85 years were included. The conditions associated with development of CRPS 1 includes lower end radius fracture, hand trauma, metacarpal fracture, soft tissue excision, carpel tunnel release and crush injury hand. All 15 patients are assessed and then explain about the procedure. Written informed consent was taken and all 15 patients were given diagnostic sympathetic block mainly stellate ganglion block. Pain intensity was evaluated before and after diagnostic block using a $10 \mathrm{~cm}$ VAS in which 0 means no pain and 10 represented most severe pain. More than 50\% reduction in VAS SCORE for at least 6 hours was considered as positive diagnostic block.

Among 18patients, 8 patients having positive stellete ganglion block, so these patients was considered for RF stellate ganglion block after 1 week and this consider as GROUP 1. 7 patients had negative stellate ganglion block so these 8 patients consider for T2-T3 sympathetic diagnostic block after one week. Among 7 patients, 3 patients having positive T2-T3 diagnostic block, so these patients was considered for RF T2-T3 sympathetic block after 1 week and this consider as GROUP 2. 4 patients had negative T2-T3 Sympathetic block, so these patients are considered as a sympathetic independent and these patients are exclude from the study. Selection of patients for RF for stellate ganglion block \& for T2-T3 sympathetic block was done after 1 week after the positive diagnostic block. In both group RF procedures were done same as diagnostic block.

All patients experienced more than 50\% pain relief after RF after 2 weeks and 1 month follow up. All patients were able to decrease their oral analgesic drugs dosage more than $50 \%$ after 1 month of RF and improved range of movement of wrist joint after 1 month of RF

No complications attritubuted to procedure were noted.

\section{Inclusion criteria}

- H/O trauma or surgery: Presence of regional pain and sensory changes following a noxious event, pain associated with finding such as abnormal skin, color, temperature changes, abnormal sudomotor activities or edema, no distribution of pain of a single nerve in extremities, The combination of these finding exceeding their expected magnitude in response to known physical damage during and following the inciting event.

- Patient had taken conservative treatment e.g. medication, physical therapy, rehabilitation programmed but failed to have pain relief.

- Any age

- Any sex 
Texila International Journal of Medicine

Volume 4, Issue 2, Dec 2016

- Patient is selected on basis of history, clinical examination, clinical diagnostic criteria and diagnostic block.

- Study is done for interventional pain management by RF, other modalities/therapies are not considered in comparison of two groups.

\section{Exclusion criteria}

- Upper limb CRPS II and lower limb CRPS.

- H/O nerve injury.

- Patient having other neuropathy like DM or other.

- Patients who has not taken conservative treatment.

- False negative block.

- Use of tobacco products or any medication that could affect sympathetic function

- Active infection at injection site

- Allergy to medication

- Previous neck surgeries, Reynaud's disease or phenomenon

- coagulopathy

Table 1. patient demographic values and etiologies

\begin{tabular}{|l|l|l|l|l|l|}
\hline No. & Age & Gender & Initial trauma & Side & $\begin{array}{l}\text { Duration } \\
\text { (weeks) }\end{array}$ \\
\hline 1 & 47 & F & Carpel tunnel release & L & 10 \\
\hline 2 & 56 & F & \# Lower end radius & L & 10 \\
\hline 3 & 48 & F & \#Lower end radius & L & 12 \\
\hline 4 & 85 & M & \#Lower end radius & R & 12 \\
\hline 5 & 52 & F & Radial distal end \# & L & 16 \\
\hline 6 & 62 & F & Carpel tunnel release & R & 22 \\
\hline 7 & 58 & M & Hand trauma & R & 26 \\
\hline 8 & 29 & M & Crush injury hand & R & 28 \\
\hline 9 & 32 & M & Metacarpal hand \# & R & 30 \\
\hline 10 & 73 & F & \#Lower end radius & L & 32 \\
\hline 11 & 43 & F & \#Lower end radius & R & 32 \\
\hline 12 & 74 & M & Metacarpal fracture & L & 33 \\
\hline 13 & 38 & F & Soft tissue release & L & 40 \\
\hline 14 & 45 & M & Hand trauma & R & 46 \\
\hline 15 & 70 & F & Radial distal end \# & R & 52 \\
\hline
\end{tabular}

\section{Results}

Table 2. vas score

\begin{tabular}{|l|l|l|l|}
\hline No. & Before block & After diagnostic SGB & After diagnostic TSB \\
\hline 1 & 7 & 2 & ----- \\
\hline 2 & 7 & 3 & ------ \\
\hline 3 & 8 & 4 & ------ \\
\hline 4 & 8 & 3 & ------ \\
\hline 5 & 8 & 4 & ------ \\
\hline 6 & 8 & 3 & ------ \\
\hline 7 & 9 & 3 & ----- \\
\hline 8 & 9 & 4 & ----- \\
\hline
\end{tabular}




\begin{tabular}{|l|l|l|l|}
\hline 9 & 8 & 6 & 3 \\
\hline 10 & 9 & 7 & 3 \\
\hline 11 & 10 & 8 & 3 \\
\hline 12 & 7 & 5 & 5 \\
\hline 13 & 9 & 6 & 6 \\
\hline 14 & 9 & 7 & 8 \\
\hline 15 & 8 & 6 & 7 \\
\hline
\end{tabular}

Table 3. shows patient's vas score rf stellet ganglionic block.

\begin{tabular}{|l|l|l|l|}
\hline PATIENT NO. & INITIAL VAS & 2 WEEKS AFTER RF & 1 MONTH AFTER RF \\
\hline 1 & 7 & 3 & 3 \\
\hline 2 & 7 & 3 & 2 \\
\hline 3 & 8 & 4 & 2 \\
\hline 4 & 8 & 3 & 2 \\
\hline 5 & 8 & 4 & 2 \\
\hline 6 & 8 & 2 & 3 \\
\hline 7 & 9 & 4 & 3 \\
\hline 8 & 9 & 3 & 3 \\
\hline
\end{tabular}

Table 4. shows patient's vas score rf t2-t3 sympathetic block.

\begin{tabular}{|l|l|l|l|}
\hline PATEINT NO. & INITIAL VAS & 2 WEEKS AFTER RF & 1 MONTH AFTER RF \\
\hline 1 & 8 & 3 & 2 \\
\hline 2 & 9 & 3 & 3 \\
\hline 3 & 10 & 3 & 1 \\
\hline
\end{tabular}




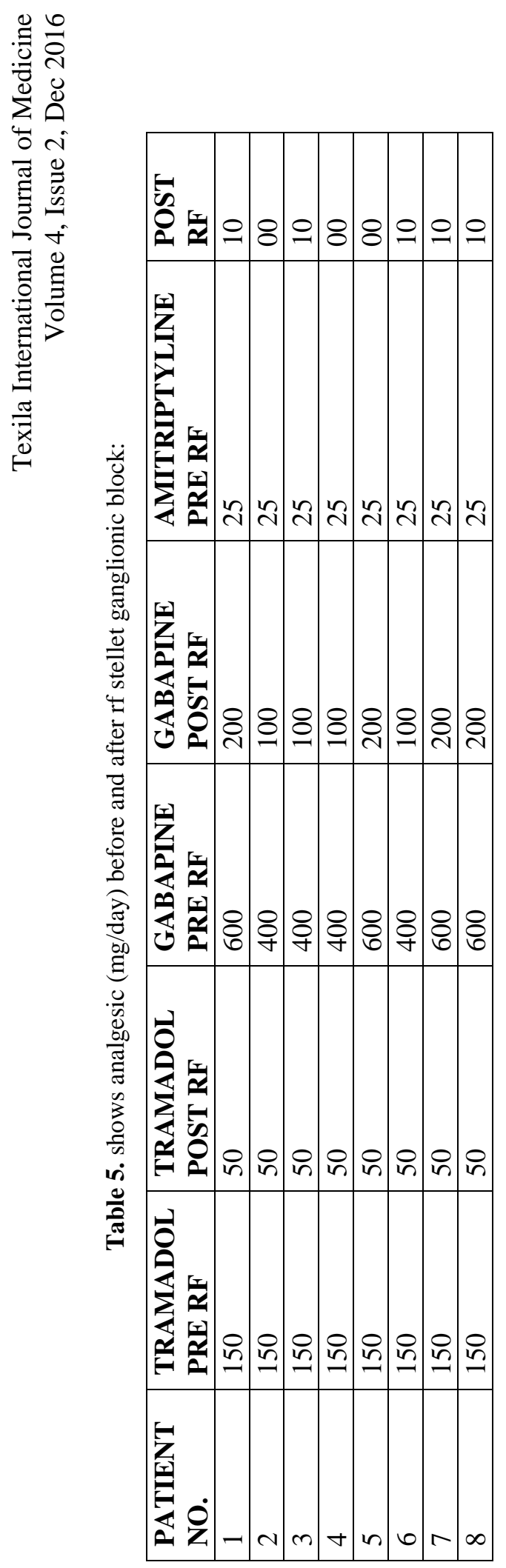




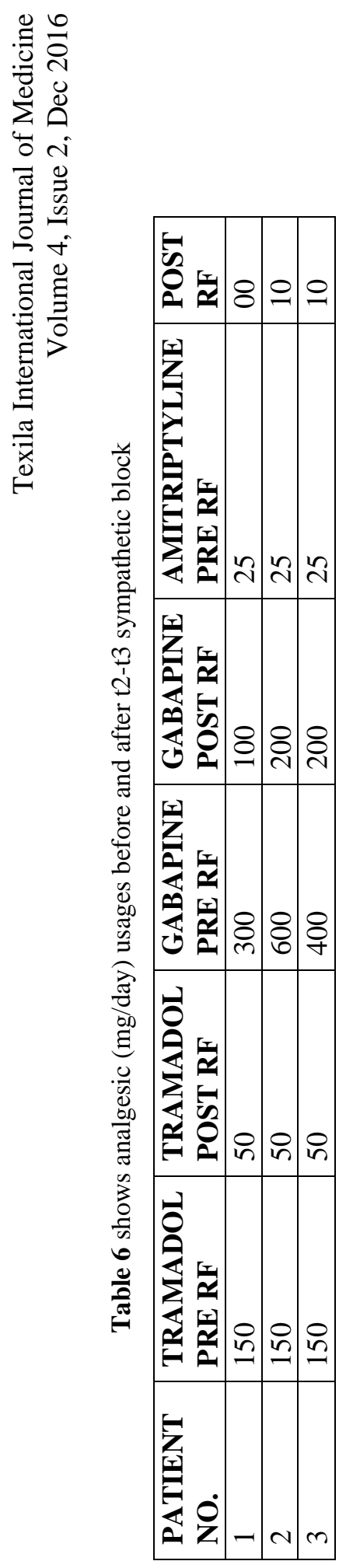


Values VAS score pretreatment and after diagnostic block are shown in table 1. Values VAS score after RF in both groups after 2 weeks and 1 month follow up are shown table 2 and table 3 . Blockade significantly improved VAS values in all patients and in both groups, however there was difference in VAS value those patients in diagnostic T2-T3 sympathetic block where diagnostic stellate ganglion block was not effective. Values of analgesic dosages before and after RF in both groups are shown in table 4 and table 5. The blockade was significantly reduced in analgesic dosages in all patients in both groups.

Though it is not included in my study, I observed that wrist movement ROM was improved in all patients that are taken in study under all review article.

Limitation:

1. Comparison between Stellate Ganglion Block and T2-T3 block is not taking in study but efficacy is taken in study.

2. False positive and false negative block should not take in consideration.

3. Negative diagnostic block not take study

4. Long term follow up more than one month is not taken in study.

\section{Discussion}

- Invasive procedure, including nerve block, spinal cord and peripheral stimulation, chemical and surgical sympathetictomy and deep brain stimulation have been used to manage CRPS type 1 . Sympathetic nervous system dysfunction is presumed to be an essential component of the syndrome and sympathetic blockage has been recommended as early as possible to interrupt and reverse the process.

- The primary goal of study was to evaluate efficacy of sympathetic block in CRPS 1 and comparison among stellate ganglion block with T2-T3 block.

- Although several studies have indicated that conventional RF is good option for long term pain relief for upper limb CRPS I. Many studies support that sympathetic block is more superior in pain relief for CRPS I than somatic block, but very few studies were done regarding efficacy between two SYMPATHETIC BLOCK for upper limb CRPS I.

- According to sympathetic pain pathway and previous studies, suggestive that many patients of upper limb CRPS 1, having “Kuntz' nerves” that leads to limitation in pain relief after successful stellate block those patients are treated with successful T2-T3 block and thus decreases false negative results.

- Therefore comparative trials of two sympathetic blocks enhance scope to see pain relief and improvement quality of life of patients.

\section{Conclusion}

Invasive procedure, including nerve block, spinal cord and peripheral stimulation, chemical and surgical sympathetictomy and deep brain stimulation have been used to manage CRPS type 1.(11) Sympathetic nervous system dysfunction is presumed to be an essential component of the syndrome and sympathetic blockage has been recommended as early as possible to interrupt and reverse the process. Sympathetic block treatment may be particularly helpful in cases in which, despite adequate doses of oral medication, pain limits a patient participation in physical and occupational therapy.

Some study suggested that stellate ganglion may not be the most suitable target for upper limb sympathetic block in CRPS 1. $(4,6,8,13)$. This suggestion is mainly due to fact that SGB may miss the sympathetic nerve fibers travelling to the upper limb in a significant proportion of individual (9). Thus by blocking T2-T3 sympathetic ganglion can cover all sympathetic fibers. In fact, Hogan(13) showed that in 100 consecutive technically well-performed SGB procedure monitored by papillary and hand temperature change, the clinically signs of upper limb sympathetic blockage were only detected after 27 of the procedure(13). $\operatorname{Kuntz}(9)$ has demonstrated that in $20 \%$ of individuals the ganglion sympathetic fibers 
Texila International Journal of Medicine

Volume 4, Issue 2, Dec 2016

projected to upper limb directly, thus bypassing the stellate ganglion after synapsing in the upper thoracic ganglia $(6,14,15)$ This is important given the major difference between TSB and SGB.

The study shows that there is chance of inadequate block after satisfactory stellate block and giving T2-T3 block among this gives good relief in such patients (19). Though posterior approach T2-T3 is more technically difficult than anterior stellate block, KUNTZ'S nerve can be reliably blocked only by a posterior approach $(14,19)$

I observed in my all study that pain score, dose of analgesic and wrist joint movement was significantly reduced in both group after RF but in comparison of both, reduction in VAS score almost similar after RF though RF T2-T3 may gives good and long term pain relief than anterior stellate block. But these require large sample size and long study period. Also CRPS is diagnosed only clinically. No laboratory test currently provides a gold standard for the diagnosis of CRPS. Following a thorough history and examination, test such as those testing for difference in skin temperature help to confirm diagnosis. But those patient having failed stellate ganglion block, T2-T3 sympathetic block gives good pain relief.

Conservative treatment like anticonvulsant and antidepressants should first line and opoids should be second line treatment to enhance pain control. Sympathetic block is more superior for pain relief in CRPS I and improve quality of life.

\section{References}

[1.] Agarwal-Kozlowski K, Lorke DE, Habermann CR, Schulte am Esch J, Beck H. Intervetnional management of intractable sympathetically mediated pain by CT guided catheter implantation for block and neuroablation of thoracic sympathetic chain: technical approach and review of 322 procedures. Anaesthesia 2011, 66. Pages 699-708.

[2.] Boas RA. Sympathetic nerve blocks: in search of a role: Reg Anasth Pain Med. 1998 May-June;23(3): 292305. Review.

[3.] Becker DE. Basic and clinical pharmacology of glucocorticosteroids. Anaesth Prog 2013 Spring; 60(1):2531; Review.

[4.] Bonica JJ, editor: The Management of Pain. Philadelphia, Lea \& Febiger, 1953 \& $4^{\text {th }}$ edition 2010.

[5.] Bonica JJ SCOTT editor, 4th edition 2010

[6.] Day M. Sympathetic blocks: the evidence Pain Pract. 2008 Mar-Apr; 8(2):98-109.

[7.] Elias M. Cervical sympathetic and stellate ganglion blocks. Pain physician. 2000. Jul; 3(3):294-304.

[8.] Fischer SG, Zuurmond WW, Birklein F, Loer SA, Perez RS. Anti-inflammatory treatment of Complex Regional Pain Syndrome. Pain 2010 Nov; 151(2) 251-256.

[9.] Goebel A. Complex regional pain syndrome in adults. Rheumatology (oxford). 2011 oct; 50(10):1739-1750.

[10.] Hogan QH, Erickson S J, Haddox JD, Abram SE. The spread of solution during stellate ganglion block. Reg. Anaesth 1992:17:78-83.

[11.] Hogan QH, Taylor ML, Goldstein M, Steven R, Kettler R. Success rates in producing sympathetic blockade by paratracheal injection. Clin J pain 1994 Jun; 10(2) 139-145

[12.] Harden RN, Oaklander AL, Burton AW, Perez RS, Richardson K, Swan M, Barthel J, Costa B, Graciosa JR, Bruehl S; Reflex Sympathetic Dystrophy Syndrome Association, Complex regional pain syndrome: practical diagnostic and treatment guidelines, $4^{\text {th }}$ edition. Pain Med. 2013 Feb; 14(2):180-229.

[13.] Kunzt A. Distribution of the sympathetic rami to the brachial plexus its relation to sympathectomy affecting the upper extremity. Arch Surg 1927:15:871-877.

[14.] Munts AG, van der Plas AA, Ferrari MD, Teeps-Twiss IM, Marinus J, Van Hilten JJ. Efficiency and safety of a single intrathecal methylprednisolone bolus in chronic complex regional pain syndrome. Eur J Pain. 2010 May;14(5):523-528.

[15.] O'Connell NE, Wand BM, McAuley J, Marston L, Moseley GL. Interventions for treating pain and disability in adults with complex regional pain syndrome. Cochrane Database Sys Rev. 2013 Apr 30;4:CD009416.

[16.] Raj PP, Lou L, Erdine S, Staats PS, Waldman SD: T2 \& T3 sympathetic nerve block and nerolysis, ch 22, pp 132-137, in Radiographic Imaging for Regional Anaesthsia and Pain Management 2003. 
[17.] Rmsaroop L, Partab P Singh B, Satyapal KS. Thoracic origine of a sympathetic supply to the upper limb: the 'nerve of Kuntz' revisited J Anat 2001:199:675-682.

[18.] Raj's practicle management of pain 4th edition 2008

[19.] Shfali A, Broatch J, RAJA SN: Web-based epidemiological survey for CRPS-1 (poster). The American Society of anaesthesiologists, New Orleans, 2005.

[20.] Yoo HS, Nahm FS, Lee PB, Lee CJ. Early thoracic sumpathetic block improves the treatment effect for upper extremities neropathic pain. Anesth Analg. 2011 Sept;113(3):605-609. 